\title{
EMERGINDO A COMPLEXIDADE DO CUIDADO DE ENFERMAGEM AO SER EM MORTE ENCEFÁLICA ${ }^{a}$
}

\author{
Emerging the complexity of nursing care facing a brain death \\ Complej idad emergente del cuidado de enfermería al paciente con muerte cerebral
}

\section{RESUMO}

0 estudo objetivou desvelar a complexidade do cuidado de enfermagem ao ser em morte encefálica. Utilizaram-se como referenciais teórico e metodológico o pensamento complexo e a Grounded Theory, respectivamente. Os dados foram coletados em um hospital universitário do nordeste brasileiro, de dezembro de 2010 a junho de 2011, por meio de entrevistas não estruturadas. A amostra teórica constituiu-se de 12 enfermeiros, distribuídos em três grupos amostrais. 0 fenômeno "Desvelando relações e interações múltiplas do ser enfermeiro na complexidade do cuidado ao ser em morte encefálica" foi delimitado por cinco categorias. Neste artigo, foi abordada a categoria "Emergindo a complexidade do cuidado de enfermagem ao ser em morte encefálica". 0 estudo evidenciou que o cuidado ao ser em morte encefálica é caracterizado por desordem e incertezas, fazendo com que o enfermeiro vivencie sentimentos diversos e ambivalentes. A sua complexidade está em compreender a sua singularidade e dialogicidade.

Palavras-chave: Cuidados de enfermagem. Morte encefálica. Enfermeiros. Cuidados intensivos. Pesquisa qualitativa.

\begin{abstract}
This study aimed to unveil the complexity of nursing care to human being in brain death. It was used as a theoretical and methodological reference, complex thinking and Grounded Theory, respectively. Data were collected in a university hospital in northeastern Brazil, from December 2010 to June 2011, through non structured interviews. The theoretical sample consisted of 12 nurses, distributed in three samples groups. The phenomenon of "Unveiling the multiple relationships and interactions to be a nurse in the complexity of care to the brain death" was delimited by five categories. In this article, was discussed the category "Emerging complexity of nursing care to be brain death". The study showed that the care facing a brain death is accompanied by disorder and uncertainties, causing the nurse to experience different feelings and ambivalent. The complexity of care facing a brain death is to understand its uniqueness and dialogical.
\end{abstract}

Keywords: Nursing Care. Brain death. Nurses, Male. Intensive care. Qualitative Research.

\section{Resumen}

Este estudio objetivó desvelar la complejidad del cuidado en enfermería al paciente con muerte encefálica. Se utilizaron como marcos teórico y metodológico el pensamiento complejo y la Grounded Theory, respectivamente. Los datos fueron recolectados en un hospital universitario en el nordeste de Brasil, entre diciembre de 2010 y junio de 2011, a través de entrevistas no estructuradas. La muestra teórica fue compuesta por 12 enfermeras asignadas en tres grupos. El fenómeno "Revelando las múltiples relaciones e interacciones en ser un enfermero en la complejidad del cuidado del paciente con muerte cerebral" fue delimitada en cinco categorías. En este artículo, se dirigió a la categoría de "Emergiendo la complejidad del cuidado de enfermería al paciente en muerte cerebral". El estudio mostró que el cuidado al ser en muerte cerebral se acompaña de desorden e incertidumbres, haciendo con que la enfermera pueda experimentar diferentes y ambivalentes sentimientos. La complejidad de los cuidados al paciente en muerte cerebral consiste en comprender su singularidad y dialogicidad.

Palabras-clave: Atención de Enfermería. Muerte Encefálica. Enfermeros. Cuidados Intensivos. Investigación Cualitativa.

\footnotetext{
${ }^{1}$ Enfermeira. Mestre em Enfermagem. Doutoranda do Programa de Pós-Graduação em Enfermagem (PEN) da UFSC. Bolsista CNPq. Membro do Grupo de Estudos e Pesquisas em Administração e Gerência do Cuidado de Enfermagem e Saúde (GEPADES). Florianópolis - SC. Brasil. Email: aline_lima_pestana@yahoo.com.br; ${ }^{2}$ Doutora em Filosofia de Enfermagem, Professora Titular do Departamento de Enfermagem e do Programa de PósGraduação em Enfermagem da UFSC, Pesquisadora 1A do CNPq, Acadêmica da ABAH, Líder do GEPADES, Florianópolis-SC. Brasil. E-mail: alacoque@newsite.com.br; ${ }^{3}$ Enfermeira. Doutora em Enfermagem. Docente do curso de graduação em Enfermagem e do Mestrado Acadêmico em Enfermagem da Universidade Federal do Maranhão. Coordenadora do Grupo de Estudo e Pesquisa na Saúde da Família, da Criança e do Adolescente GEPSFCA. Membro do GEPADES. São Luís-MA. Brasil. Email: fgeorginasousa@hotmail.com
} 


\section{INTRODUÇÃO}

0 paciente em morte encefálica (ME) é definido como um ser que apresenta parada total e irreversível do cérebro e tronco cerebral, mas que mantém, temporária e artificialmente, a função cardiorrespiratória ${ }^{1}$. Somente 1 a $4 \%$ das pessoas que morrem no hospital e entre 10 e $15 \%$ daquelas que morrem em Unidades de Terapia Intensiva (UTI) apresentam o quadro de ME, sendo consideradas potenciais doadoras de órgãos ${ }^{2}$. 0 traumatismo cranioencefálico, 0 acidente vascular encefálico e a lesão cerebral hipóxico-isquêmica são responsáveis por quase $90 \%$ das causas da $\mathrm{ME}^{3}$.

No ano de 1997, o Conselho Federal de Medicina (CFM) publicou as normas para o diagnóstico de ME no Brasil. Os critérios clínicos devem ser registrados no termo de declaração de ME por dois médicos experientes, não necessariamente neurologistas, em intervalo que varia conforme a faixa etária de cada paciente. É necessária a realização de exame complementar que demonstre ausência de atividade elétrica cerebral ou ausência metabólica cerebral, ou ausência de perfusão sanguínea cerebral. Essa normatização permitiu a doação de órgãos e a exigência de cuidados intensivos para manter o paciente como potencial doador ${ }^{1}$.

0 cuidar do paciente em ME, na maioria das vezes, ainda está vinculado ao modelo biomédico, influenciado pelo paradigma cartesiano, que o vê como um ser morto, sem perspectiva de vida, que consequentemente não necessita de cuidados específicos e intensivos. No entanto, com o aprimoramento tecnológico na área de transplantes de órgãos, percebeu-se que o paciente nesta condição começou a ser mais bem cuidado graças à possibilidade de doação de órgãos e tecidos, que permitiu que outras pessoas pudessem recomeçar suas vidas com mais qualidade.

Desse modo, é importante conduzir adequadamente 0 potencial doador de órgãos com o mesmo empenho e dedicação que qualquer outro paciente da UTI. Esse paciente não deve ser visto como um ser mor to que não necessita de cuidados; deve-se avançar no olhar reconhecendo-o para além de um corpo. Não "deve deixar de ser singular e transformar-se em um objeto" ", mas ser visto em sua totalidade e na condição que lhe é própria de ser humano.

Partimos da hipótese de que cuidar do ser em ME envolve o princípio dialógico que permite assumir racionalmente a inseparabilidade de duas noções contraditórias, ou seja, une dois princípios que tendem a se excluir, entendendo-os como simultaneamente concorrentes, antagônicos e complementares ${ }^{5}$. Isso pode ser percebido na relação de cuidado estabelecida com o paciente em ME, que congrega em si vida e morte, não como dicotomia, mas como uma unidade complexa.
Diante do exposto, questiona-se: Que significados os enfermeiros atribuem ao cuidado ao paciente em morte encefálica? 0 presente estudo teve como objetivo desvelar a complexidade do cuidado de enfermagem ao ser em morte encefálica.

\section{METODOLOGIA}

0 estudo é um recorte da dissertação intitulada "Desvelando relações e interações múltiplas do ser enfermeiro na complexidade do cuidado ao ser em morte encefálica na Unidade de Terapia Intensiva", ${ }^{6}$ que buscou compreender os significados do cuidado ao paciente em morte encefálica para enfermeiros de um hospital universitário, construindo um modelo teórico.

Desse modo, buscou-se explorar, na perspectiva do princípio dialógico do pensamento complexo ${ }^{5}$, o componente do modelo paradigmático: Emergindo a complexidade do cuidar ao Ser em ME. 0 pensamento complexo estimula a desconstrução de padrões estabelecidos ao longo dos tempos sobre o paciente em ME, possibilitando o desenvolvimento de olhares mais profundos sobre este ser.

Trata-se de uma pesquisa qualitativa, tendo como referencial metodológico a Grounded Theory ou Teoria Fundamentada nos Dados (TFD). Consiste em um modo de compreender fenômenos que são descobertos, desenvolvidos conceitualmente e estabelecidos por um processo de coleta e análise dos dados sistematicamente conduzidos ${ }^{7}$. Visa compreender a realidade a partir da percepção ou significado que certo contexto ou objeto tem para a pessoa, gerando conhecimentos, aumentando a compreensão e proporcionando um guia significativo para a ação8.

A coleta de dados foi realizada com enfermeiros de um Hospital Universitário do nordeste brasileiro, no período de dezembro de 2010 a junho de 2011; a técnica de coleta foi a entrevista não estruturada, sendo esta nor teada pela seguinte pergunta: 0 que significa para você cuidar do paciente em morte encefálica? No decorrer da entrevista, outras questões foram formuladas para aprofundar a compreensão da experiência em cuidar do paciente em ME.

Para a seleção dos participantes buscaram-se locais, pessoas ou fatos que potencializassem oportunidades de descobrir variaç̃̃es entre conceitos e de tornar densas categorias em termos de suas propriedades e de suas dimensões ${ }^{8}$. 0 tamanho da amostra teórica foi determinado pela Saturação Teórica dos dados, conforme preconiza a TFD, sendo atingida com 12 entrevistas. Alcança-se a saturação quando não surgem novos dados importantes em relação a uma determinada categoria; quando essa está bem desenvolvida nas suas propriedades e dimensões; e quando as relações entre as categorias estão bem estabelecidas e validadas 8 .

A intenção no início da coleta de dados era entrevistar apenas os enfermeiros que atuavam em UTI. Porém, no 
desenvolvimento da pesquisa, a partir da análise dos dados (codificação das entrevistas, memorandos e diagramas) e das hipóteses construídas ao longo desse processo, surgiu a necessidade de se buscar outros participantes, constituindo assim outros grupos amostrais para a compreensão do fenômeno. Dessa forma, os participantes foram organizados em três grupos amostrais. 0 primeiro grupo amostral foi composto por quatro enfermeiras assistenciais da UTI do hospital universitário que atenderam ao critério de inclusão de ter vivenciado a experiência de cuidar de um paciente em ME. Os participantes tinham idades entre 24 e 39 anos e atuavam no referido setor entre 1 e 7 anos.

0 segundo grupo amostral foi composto por quatro enfermeiros que desenvolviam suas atividades no serviço de captação de órgãos e transplante do referido hospital, que estavam na faixa etária de 25 a 49 anos e tinham tempo de experiência na UTI de 6 meses a 19 anos. As hipóteses resultantes do primeiro grupo amostral apontaram que 0 cuidado ao paciente em ME é complementado/compartilhado com o serviço de captação de órgãos e transplante. Dessa forma, a questão nor teadora para entrevista com este grupo foi: como se dá o processo de complementaridade do cuidado a este paciente? Como estão organizadas as práticas de cuidado ao paciente em morte encefálica?

Os enfermeiros do segundo grupo amostral referiram que, com a evolução do conhecimento científico na área de transplante, houve modificação no cuidado ao paciente em ME. Desse modo, considerou-se importante buscar a compreensão de como era o cuidado ao paciente em ME antes da consolidação das políticas de transplante. Assim, foram entrevistados quatro enfermeiros que haviam trabalhado na UTI em período anterior à implantação do serviço de transplante no referido hospital; a questão da entrevista foi: Como era o cuidado ao paciente em morte encefálica quando você trabalhava na UTI? Como foi a sua experiência em cuidar deste paciente?

As entrevistas foram gravadas, transcritas e posteriormente reapresentadas aos participantes do estudo no intuito de permitir que os dados fossem validados por eles, para correções e acréscimos, caso julgassem necessários. A análise dos dados ocorreu de modo simultâneo a coleta, seguindo as etapas propostas pela TFD: codificação aberta, axial e seletiva.

Na codificação aberta, os dados foram analisados linha por linha com o objetivo de identificar cada ideia/incidente/ evento. A partir disso, foi dado um nome, formando os códigos preliminares. Em seguida, estes foram reunidos, por similaridades e diferenças, dando oportunidade de elaborar os códigos conceituais ou conceitos. Na codificação axial, os dados foram reagrupados, visando relacionar categorias às suas subcategorias, no sentido de obter uma explicação mais clara e completa sobre o fenômeno, assim como as suas propriedades e dimensões. Na fase da codificação seletiva, as categorias e subcategorias encontradas anteriormente foram comparadas e analisadas continuamente com o objetivo de integrar e de refinar a teoria, fazendo emergir dessa forma a categoria central ou 0 fenômeno, para assim desenvolver a explicação teóricå.

Para classificar e organizar as associações emergentes entre as categorias foi utilizado um esquema organizacional, denominado paradigma ou modelo paradigmático. Trata-se de uma estrutura analítica que ajuda a reunir e a ordenar os dados sistematicamente, de forma que estrutura e processo sejam integrados. Esse modelo estabelece uma relação entre as categorias a partir dos seguintes componentes: fenômeno, contexto, condições causais e intervenientes, estratégias e as consequências ${ }^{8}$.

A partir do processo de análise foi construído o fenômeno "Desvelando relações e interações múltiplas do ser enfermeiro na complexidade do cuidado ao ser em morte encefálica" sustentado pelas seguintes categorias: Cuidando do ser em ME em Unidade de Terapia Intensiva (contexto); Tendo que organizar as práticas de cuidado em UTI ao ser em ME (condição causal); Considerando as interveniências facilitadoras e dificultadoras do cuidado ao ser em ME na perspectiva das relações/interações entre o ser enfermeiro, ser equipe e família (condição interveniente); Incorporando atitudes para cuidar da complexidade do ser em ME (Estratégia) e Emergindo a complexidade do cuidado de enfermagem ao ser em ME (Consequência). Devido à importância e relevância desta última, optou-se, para esse manuscrito, por abordá-la separadamente, juntamente com suas respectivas subcategorias.

A validação do modelo paradigmático foi efetivada com uma enfermeira especialista em Terapia Intensiva e com uma pesquisadora expertise em Teoria Fundamentada nos Dados.

0 estudo seguiu as recomendações da Resolução 196/ 96 do Conselho Nacional de Saúde ${ }^{9}$, recebendo parecer favorável do Comitê de Ética em Pesquisa do Hospital Universitário onde o estudo foi realizado, sob o protocolo 3936/2010. A obtenção da anuência dos participantes se deu por meio da assinatura do Termo de Consentimento Livre e Esclarecido. Para preservar o anonimato dos participantes, seus nomes foram substituídos por um código, ou seja, pela letra "E" seguida do número de ordem da entrevista (E1, E2, E3...E12).

\section{RESULTADOS}

"Emergindo a complexidade do cuidado de enfermagem ao ser em ME" como consequência do modelo paradigmático construído está composta por uma categoria e três subcategorias que inter-relacionadas evidenciam que o cuidar do Ser em ME permite emergir reações e variados sentimentos no enfermeiro, revelando a complexidade do cuidado a esse paciente, que exige trabalho em equipe e multidisciplinar em conhecimentos, ações e intervenções que se articulam entre si e se complementam.

Essa categoria é composta por três subcategorias: "Emergindo os sentimentos e reações do ser enfermeiro no processo de cuidado ao ser em ME", "Apontando a complexidade 
do cuidado ao ser em ME" e "Avançando nas práticas de cuidado ao ser em ME". A primeira subcategoria descreve que no processo de cuidado ao ser em ME emergem reações e sentimentos ambivalentes e diversos nos enfermeiros, mesmos naqueles com anos de experiência em UTI, o que pode ser evidenciado nas falas:

Eu me sinto numa transição de emoções. Eu me sinto triste por saber que de qualquer maneira o paciente em ME não vai ter retorno [...] A tristeza existe por mais que a gente trabalhe por anos a fio [...] Quando ele é para ser doador e não dar certo, realmente a frustração acontece. Equando ele não é doador de qualquer maneira nos sentimos gratificadas porque estamos ali cuidando dele (E1).

A tristeza em cuidar do paciente em ME é saber que estava perdendo-o (E11).

Outros sentimentos que emergem são a decepção e a frustração, principalmente se o ser em ME é potencial doador e ocorre o óbito, interrompendo o processo de doação de órgãos, ou quando é vivenciada a falta de recursos para prover o cuidado:

O processo do cuidado ao paciente potencial doador em morte encefálica que evolui a óbito é frustrante porque até então ele está sob os nossos cuidados. Equeremos que tudo dê certo [...] Mas, quando o processo de doação não acontece ou foi interrompido ali mesmo na UTI, é decepcionante (E1).

Agora, quando eu não tenho as coisas para cuidar do paciente em ME ao meu alcance eu fico muito frustrada (E7).

Além de sentimentos de tristeza e frustração, emergem também nos enfermeiros a dúvida e a confusão em saber se o paciente em ME está realmente morto:

Apesar de o paciente estar em ME, ainda há uma "vida" ali. Ainda tem condução cardíaca, tem aquele pulso final que ainda está ali. Então, "ainda é uma vida que está ali". Mesmo que seja pela ventilação mecânica, pela medicação que está entrando [...] Porque você tem que cuidar da vida, você foi instruído para cuidar da vida, mas ali fica aquela dúvida: é morte? Não é? (E3).

As reações mais frequentes apresentadas pelos enfermeiros foram estresse, sofrimento, choro e sufoco por ter suas emoções reprimidas na UTI, como demonstradas nas falas seguintes:
Não temos condições de exprimir ou ter aquele tempo para se emocionar, digo, chorar, lamentar e tudo [...] Não choro, mas talvez eu chore saindo de lá do ambiente de trabalho, pensando nas coisas que vemos. Lá realmente não dá para chorar porque é tanta coisa para ser feita e nós acabamos nos detendo naquelas atividades (E1).

Eaítem o estresse do processo de cuidado porque na ME tudo é muito rápido (E2).

Na subcategoria "Apontando a complexidade do cuidado ao ser em ME" evidenciou-se, a partir da percepção dos enfermeiros, que a complexidade do cuidado está atrelada à mudança na concepção do Ser em ME que não demandava muitos cuidados mas atualmente exige cuidados de paciente grave, principalmente nos casos de potencial doador de órgãos.

Porque passou de um paciente que não dava muito trabalho para um paciente que está dando muito trabalho [...] É um paciente que requer muitos cuidados. Houve uma mudança na concepção do paciente em ME (E5).

Aliada à mudança de concepção do ser em ME, o cuidar desse paciente é permeado pelas dimensões técnico-científica e humana. A dimensão técnico-científica é constituída, na visão dos enfermeiros, pelas habilidades, protocolos e tecnologias empregadas no cuidado. 0 objetivo dessa dimensão é cuidar do ser em ME com todo suporte tecnológico e científico, visando manter a estabilidade hemodinâmica. Na dimensão humana, 0 enfermeiro busca valorizar, acolher e apoiar a família do ser em ME com a finalidade de cuidar com dignidade e respeito dos participantes deste processo de cuidado. Os enfermeiros referem que estas dimensões são complementares, como pode ser observado nas falas a seguir:

A dimensão técnico-cientifica são as habilidades. É lidar com o paciente em ME como se fosse um paciente grave. O paciente em ME é um paciente grave como qualquer outro aqui da UTI. Ele tem as suas peculiaridades, mas ele é um paciente grave e precisa ser cuidado com o mesmo esmero, mesma dignidade, com o mesmo saber, com a mesma habilidade que a gente teria cuidando de outro. [...] Na dimensão humana vamos procurar acolher mais essa família. [...] cuidar do paciente em ME com respeito. Tratá-lo como ser humano, como ser doente que precisa do nosso cuidado. Eu acredito que as dimensões técnico-cientifica e humana se completam no processo de cuidar do paciente em ME (E4). 
0 cuidado ao ser em ME exige um trabalho em equipe, complementar entre a equipe da UTI e a do serviço de captação de órgãos e transplante. Na percepção dos enfermeiros do serviço de captação de órgãos e transplante, a complementaridade do cuidado évista como "somar com a equipe da UTI", "fazer com a equipe da UTI", "providenciar os cuidados ao paciente em ME", "é um processo" que exige a interdependência profissional, cooperação, integração da equipe multiprofissional e boa comunicação, como descrito nas falas a seguir:

O nosso complementar é justamente esse somar, chegar junto, fazer [...] Sempre você tem que chegar fazendo juntamente com a equipe e nunca chegar mandando. Tem que ajudar, fazer (E5).

Lembrando sempre que é importante manter uma boa comunicação com os profissionais. Essa é uma ferramenta considerada indispensável (E8).

Na subcategoria "Avançando nas práticas de cuidado ao ser em ME", os enfermeiros referem que os pacientes em ME potenciais doadores são transferidos ao Hospital Universitário, e, deste modo, os enfermeiros da UTI vão modificando e aperfeiçoando a prática profissional. Com a experiência de cuidar do ser em ME, o enfermeiro adquire crescimento profissional e compreende que esse paciente também precisa de cuidados. Dessa forma, existe mudança na postura do profissional diante desse paciente, ou seja, assume mais o compromisso de cuidálo, como pode ser evidenciado nas falas a seguir:

Hoje em dia, os profissionais assumem um compromisso maior com esse doador, pelo menosé o que eu vejo aqui dentro desse hospital, principalmente a enfermagem. Acredito que seja por conta da prática, porque a maioria dos pacientes em ME vem para este hospital (E6).

Então, o que mudou para mim, como enfermeira hoje, é mais na questão de saber como lidar com a situação de ME, ter o entendimento de que aquela pessoa [ser em ME] precisa do meu cuidado também (E2).

Além da mudança na postura do profissional, existe modificação na concepção do cuidado ao ser em ME. Antes, o paciente em ME era deixado de lado da assistência e nenhum cuidado específico era dirigido a ele, permanecendo na UTI até a parada cardiorrespiratória, como evidenciado na fala:

Ao longo do tempo a gente associou que o paciente em ME era aquele paciente que ficava de lado da assistência, esperando ele parar. Hoje, [...] o paciente em ME tem que ser tratado igual aos outros pacientes graves. De grave para gravíssimo. Porque a cada hora que passa, o paciente em ME fica mais grave, vai complicando [...] Houve uma mudança na concepção do paciente em ME (E5).

0 enfermeiro reconhece e identifica as modificações ocorridas tanto na concepção do ser em ME quanto no processo de cuidar do mesmo. No entanto, deseja práticas de cuidado que atendam a toda a complexidade do cuidado, vislumbrando melhores resultados.

Quero muito que as nossas práticas com o paciente em ME mudem, cresçam, melhorem e sejam mais efetivas (E7).

\section{DISCUSSÃO}

0 processo de cuidado ao ser em ME potencial doador ocorre na UTI, e é caracterizado por desordem e incertezas, fazendo com que o enfermeiro vivencie sentimentos diversos e ambivalentes. Um estudo realizado com o objetivo de compreender a vivência da equipe de enfermagem em relação aos cuidados de manutenção de potenciais doadores constatou que as reações e sentimentos da equipe diante da morte encefálica foram de insegurança, incapacidade, constrangimento, impotência, sofrimento e dor ${ }^{10}$.

Em outro estudo, a ME despertou sentimentos de tristeza, angústia, medo e frustração em enfermeiros que trabalhavam em captação de órgãos para transplante. A frustração foi desencadeada nestes profissionais, pois estabeleciam vínculo com o ser em ME potencial doador e também identificavam-se com este paciente pela sua condição humana. Destaca-se ainda que o enfermeiro cuida do paciente em ME, ser morto, na perspectiva de gerar vida por meio da doação ${ }^{11}$. No entanto, quando ocorre a parada cardíaca, o enfermeiro sente-se frustrado, pois o objetivo do cuidado de enfermagem não foi alcançado.

A morte provoca angústia, horror e dor. "A dor provocada por uma morte só existe se a individualidade do morto estiver presente e reconhecida: quanto mais o morto for próximo, [...], mais violenta é a dor" (12:32). Acrescenta-se que a perda do ser em ME é sentida com mais intensidade quando são estabelecidos vínculos cuidativos e afetivos entre o paciente em ME e o enfermeiro. Estes profissionais sentemse frustrados e apresentam dificuldades para aceitar e lidar com a morte do paciente ${ }^{13}$.

A dúvida, a confusão e a incerteza são sentimentos constituintes do processo de cuidado do ser em ME. Em um estudo desenvolvido com enfermeiros de três hospitais suecos foi identificado que a dúvida dos profissionais consistia em saber se a pessoa que estava em ME 
encontrava-se realmente morta, pois desconfiavam dos testes para diagnosticar a ME, devido ao pouco conhecimento de como eram realizados ${ }^{14}$.

0 conceito de morte encefálica encontra resistência e gera sentimentos de dúvidas e incertezas, não somente na população, mas também entre os profissionais de saúde que cuidam do potencial doador ${ }^{15}$. Estes sentimentos são explicados pelo fato de o Ser em ME apresentar em si, simultaneamente, elementos próprios de quem está vivo e que também sustentam a sua morte ${ }^{16}$. Outro fator que contribui para isso é que o profissional de saúde não está sendo preparado durante a formação para lidar com 0 paciente em ME potencial doador ${ }^{17}$.

Para o enfermeiro conviver e cuidar do ser em ME, é necessário compreendê-lo como um ser singular e diferente, ampliando o campo de visão em relação a este ser, enxergando-o como um paciente grave e estabelecendo sentido às interações de cuidado.

A complexidade do cuidado ao ser em ME permite ao enfermeiro experienciar a singularidade e a adversidade, a compreensão e a incompreensão, o real e o utópico, a vida e a morte. É necessário, portanto, articular um cuidado pautado em diferentes saberes, a fim de valorizar e potencializar o conhecimento interdisciplinar, caminhando na direção de uma vivência/assistência complexa, plena e flexível e, consequentemente, de um paradigma de humanização, no qual o paciente em ME é apreendido não pela sua possibilidade de doação, mas pela singularidade que o torna um SER especial e único no universo.

A complexidade do cuidado de enfermagem ao ser em ME é permeada por duas dimensões: a técnico-científica e a humana. Em um estudo desenvolvido em UTI foi possível identificar as dimensões dos cuidados intensivos; foram encontrados o cuidado como prática assistencial e o cuidado como relação dialógica. 0 primeiro incluía a execução de procedimentos técnicos e apoio ao cliente na sua integralidade como ser complexo, bem como envolvia o compromisso e a responsabilidade na relação de cuidado ${ }^{18}$, aproximando-se com a dimensão técnico-científica.

0 cuidado como relação dialógica foi evidenciado a partir da intenção do cuidador em ser autêntico, estar presente e ser capaz de experienciar um mundo subjetivo de cuidado com o outro, valorizando não somente os aspectos técnico-científicos ${ }^{17}$, mas o ser humano, mesmo que este esteja morto.

Os participantes deste estudo referiram que houve avanço nas práticas de cuidado ao ser em ME. Isso se relaciona com a evolução do conhecimento científico na área de transplante e a criação e implantação de uma legislação própria para a doação de órgãos e transplantes, o que proporcionou modificação na concepção tanto do paciente em ME quanto do cuidado a este, passando a ser mais valorizado em sua multidimensionalidade?

\section{CONCLUSÃO}

A categoria "Emergindo a complexidade do cuidado de enfermagem ao ser em ME" configura-se como a consequência do modelo paradigmático intitulado "Desvelando relações e interações múltiplas do ser enfermeiro na complexidade do cuidado ao ser em morte encefálica". É composta pelas subcategorias: Emergindo os sentimentos e reações do ser enfermeiro no processo de cuidado ao ser em ME, Apontando a complexidade do cuidado ao ser em ME e Avançando nas práticas de cuidado ao ser em ME.

0 desvelar da complexidade do cuidado de enfermagem ao ser em ME implica promover uma mudança de paradigma na forma como este ser é visto e cuidado. Significa superar, indo muito além do paradigma reducionista que permite enxergá-lo como um morto. Significa incorporar um novo modo de cuidar, uma nova consciência, que transpõe o reducionismo de que este paciente não precisa de cuidados.

0 pensamento complexo propõe um olhar multidimensional para o ser em ME, permitindo ao enfermeiro refletir e desconstruir as barreiras do cuidar tradicional e hegemônico. Pressupõe atribuir um novo significado às práticas de cuidado que visualize a complexidade das relações cuidativas com o ser em ME, possibilitando emergir a importância do cuidado a este paciente, compreendendo a sua singularidade e dialogicidade.

Apontam-se necessidades de revisão no currículo dos cursos de graduação em Enfermagem com o objetivo de ampliar o conhecimento sobre o conceito e o protocolo diagnóstico de ME, otimizando o processo de doação de órgãos, no intuito de minimizar sentimentos de incertezas e dúvidas ao cuidar do ser em ME, preparando os profissionais de saúde para atuação eficaz no campo da doação de órgãos e tecidos.

\section{REFERÊNCIAS}

1. Conselho Federal de Medicina.[BR] Resolução $n^{0} 1.480$, de 08 de agosto de 1997. Estabelece os critérios para caracterização de morte encefálica. Diário Oficial da Republica Federativa do Brasil, Brasilia, DF, 21 ago 1997. Seção 1: 18.227- 228.

2. Garcia VD. A política de transplantes no Brasil. Rev AMRIGS, Porto Alegre, 2006 out/dez; 50 (4): 313-20.

3. Morato EG. Morte encefálica: conceitos essenciais, diagnóstico e atualização. Revista Medica de Minas Gerais. 2009; 19 (3): 227-36.

4. Nascimento ERP, Trentini M. 0 cuidado de enfermagem na Unidade de Terapia Intensiva -UTI: teoria humanística de Paterson e Zderad. Rev Latino-Am Enfermagem, Ribeirão Preto, 2004 mar/abr; 12 (2): 250-57. 
5. Morin E. A cabeça bem-feita: repensar a reforma, reformar 0 pensamento. $15^{\mathrm{a}}$ ed. Rio de Janeiro: Bertrand Brasil; 2008.

6. PestanaAL. Desvelando relações e interações múltiplas do ser enfermeiro na complexidade do cuidado ao ser em morte encefálica na Unidade de Terapia Intensiva [dissertação]. Florianópolis: Universidade Federal de Santa Catarina; 2011

7. Sousa FGM. Tecendo a teia do cuidado à criança na atenção básica de saúde: dos seus contornos ao encontro com a integralidade. [tese]. Florianópolis: Universidade Federal de Santa Catarina; 2008.

8. Strauss A, Corbin J. Pesquisa qualitativa: técnicas e procedimentos para o desenvolvimento de teoria fundamentada. $2^{\mathrm{a}}$ ed. Porto Alegre: Artmed; 2008.

9. Conselho Nacional de Saúde (BR). Resolução nº 196, de 10 de outubro de 1996. Aprovar diretrizes e normas reguladoras de pesquisas envolvendo seres humanos .Inf Epidemiol SUS. 1996; 5 (2): 14-41.

10. Lemes MMD, Bastos MAR. Os cuidados de manutenção dos potenciais doadores de órgãos: estudo etnográfico sobre a vivência da equipe de enfermagem. Rev Latino-Am Enfermagem. 2007 set/out; 17(6): 98691.

11. Lima AAF, Silva MIP, Pereira LL. Sofrimento e contradição: o significado da morte e do morrer para enfermeiros que trabalham no processo de doação de órgãos para transplante. Enferm Global. [on-line] 2009 fev; 15.

12. Morin E. O homem e a morte. Tradução de Cleone Augusto Rodrigues. Rio de Janeiro: Imago; 1997.

13. Backes MTS, Erdmann AL, Büscher A, Backes DS. Desenvolvimento e validação de teoria fundamentada em dados sobre o ambiente de unidade de terapia intensiva. Esc Anna Nery. 2011 dez; 15(4): 769-75.

14. Flodén A, Forsberg A. A phenomenografic study of ICU-nurses's perceptions of and attitudes to organ donation and care of potencial donors. Intensive Crit Care Nurs. 2009 dez; 25 (6): 306-13.

15. Moraes EL, Massarollo MCKB. Recusa de doação de órgãos e tecidos para transplante relatados por familiares de potenciais doadores. Acta Paul Enferm. 2009; 22(2): 131-35.

16. Vargas MA, Ramos FRS. A morte cerebral como presente para a vida: explorando práticas culturais contemporâneas. Texto \& Contexto Enferm. 2006 jan./mar; 15(1): 137-45.

17. White G. Intensive care nurses' perceptions of brain death. Aust Crit Care. 2003 Feb; 16(1): 7-14.

18. Nascimento KC, Erdmann AL. Understanding the dimensions of intensive care: transpersonal caring and complexity theories. Rev Latino-Am Enfermagem. $2009 \mathrm{abr} ; 17$ (2): 215-21.

\section{NOTAS}

aArtigo elaborado a partir da dissertação intitulada "Desvelando relações e interações múltiplas do ser enfermeiro na complexidade do cuidado ao ser em morte encefálica na Unidade de Terapia Intensiva", desenvolvida no Programa de Pós-Graduação em Enfermagem (PEN) da Universidade Federal de Santa Catarina (UFSC), 2011. 